\title{
THE EXPERIMENTAL INVESTIGATION OF THE SCREEN OPERATION IN THE PARAMETRIC RESONANCE CONDITIONS
}

\author{
Łukasz BAKK$K^{*}$, Stanisław NOGA*, Feliks STACHOWICZ* \\ *Faculty of Mechanical Engineering and Aeronautics, Rzeszow University of Technology, \\ Al. Powstańców Warszawy12, 35-959 Rzeszów, Poland \\ lbak@prz.edu.pl, noga@prz.edu.pl, stafel@prz.edu.pl
}

received 15 May 2015, revised 10 December 2015, accepted 11 December 2015

\begin{abstract}
In this paper the experimental studies of the screen working in the parametric resonance condition are discussed. The investigations are conducted for laboratory parametric resonance screen. The measuring test is performed for four cases of tension force values. The full sheet metal instead of the sieve is used. For each considered case the natural frequency of the plate and the parameter modulation frequency are determined. The achieved results are presented and discussed. It is shown that the highest sieve plate amplitude is obtained when the parameter modulation frequency is two times larger than natural frequency of the sieve plate. This parametric resonance vibration was observed only for tension force equal to $4000 \mathrm{~N}$ because of the rotational speed limits of electrical vibratos.
\end{abstract}

Keywords: Vibrating Screen, Parametric Resonance, Natural Frequencies

\section{INTRODUCTION}

Vibro-impact mechanisms, such as screeners and drilling/cutting devices, are usually designed to operate in a resonance regime (RR) (Michalczyk, 1995). Then the most powerful energy flux can be achieved from the exciter to the vibrating component, and high oscillation amplitude of the target structure can be obtained using excitation of a relatively low intensity. Parametric resonance $P R$ is the state of oscillating instability which can arise under periodic disturbance of a parameter of the structure, for example, under periodic variation of the pendulum length or the tensile force in a beam, plate or a flexible screen (Parszewski, 1992; Virgin, 2007).

The problem of the parametric resonance in mechanical systems is studied by numerous researches and development centres (Başar et al., 1987; Bąk et al., 2013; de Silva, 2005; Hagedorn and Koval, 1971; Michalczyk, 1995; Nguyen, 2013; Osiński, 1985; Parszewski, 1982; Slepyan and Slepyan, 2013, 2014). The parametric resonance phenomenon has been known since 19th century. For the first time, this phenomenon is described by Hill and Mathieu and can be found as the fundamental theory related to the vibration of the slender structures and the parametric resonance phenomenon (the so-called Hill or Mathieu equation (de Silva, 2005; Kaliski, 1996; Parszewski, 1982; Virgin, 2007)). The analysis of parametric vibration in the beam system including the Timoshenko theory is discussed by Hagedorn and Koval (1971).While the parametric vibration of a Bernoulli beam with constant transverse loading is analysed by Osiński (1985).In paper of Yang and Chen (2006), the problem of stability in transverse parametric vibration of axially accelerating beam is studied. In the consideration the Newton's second law and Boltzmann's superposition principle are used. The numerical method, based on a finite element discretisation, is proposed to solve the parametric resonance problem of a shell structures by Başar et al. (1987).
Work of Nguyen (2013) deals with analysis of the parametric resonance of the simple supported rectangular plate by using the von Karman large-deflection theory. In general parametric resonance is undesirable effect in machines construction (for example in gear wheels cooperation, slender structures, and other) (Virgin, 2007). But in some cases, the parametric resonance phenomenon, allows to achieve higher performance processing.

A good example of confirmation, can be vibratory machines for screening and transport of aggregate (Bak et al., 2013; Osiński, 1985). Free vibrations of the screen sieve, operation in the parametric resonance conditions, are analyzed in the paper of Bak et al. (2013) by using analytical and numerical method. The simple dynamic model of the vibrating screen operating in the parametric resonance mode is proposed by Slepyan and Slepyan $(2013,2014)$. The proposed model includes two mass points connected by the prestressed string. Furthermore, in the above mentioned papers the description of the sample screen system working in the parametric resonance condition is presented. While in papers of Song et al. (2009) and Li (1995), new constructions of the vibrating screen working near resonance zone and chaotically excited are considered. Complex vibration analysis of the new type of large vibrating screen with hyperstatic net-beam structure is conducted in paper of Zhao et al (2009). Another analysis related to the motion of the sieve of the vibrating screen along the variable elliptical trace during screening processes is discussed by He and Liu (2009). This paper continues the recent authors investigations concerning the vibration of screen systems (Bak et al., 2013). The present paper deals with the experimental investigations of the laboratory screen operating in the parametric resonance condition. The aim of these investigations is to determine the natural frequencies of the sieve and the parameter modulation frequencies (the excitation frequencies) as a function of the sieve tension force. Firstly, the description of the screen is presented. Then the results of the dynamical investigations are presented and discussed. 


\section{DESCRIPTION OF THE LABORATORY SCREEN}

The laboratory parametric resonance screen (Fig.1) is made in accordance to the project based on the GEPARD-2 screen. GEPARD-2 screen was formerly designed by V. Slepyan from Loginov Partnership Mining Company in Kiev. All assemblies of the screen are mounted on the base frame which is composed of square profiles welded together.

The laboratory screen consists of the following assemblies and devices:

- sieve size: $700 \times 750 \mathrm{~mm} \times 1 \mathrm{~mm}$,

- two electrical vibrators with eccentric mass control from 0 to $2.97 \mathrm{kN}$,

- four strain gauge sleeves connected with HBM QUANTUM X840 data acquisition system,

- two PCB model 353B14 accelerometer,

- laser speedometer with resolution $1 \mathrm{rpm}$,

- NI 9234 IEPE measuring module supported by the Signal Express software.

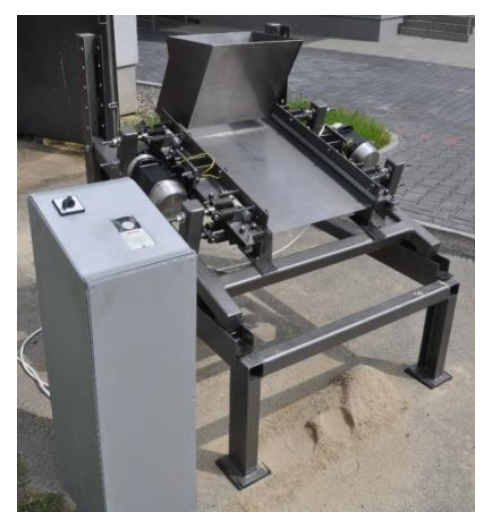

Fig. 1. Parametric resonance screen

Moreover in the experiment the PHANTOM V710 high speed camera is used. The total estimated weight of the screen system is $200 \mathrm{~kg}$. In the presented investigations, the full sheet metal instead of the sieve is used. For each case the value of the tension force was established with using strain gauges sleeves which are mounted on the suspension bolt (Fig.2).

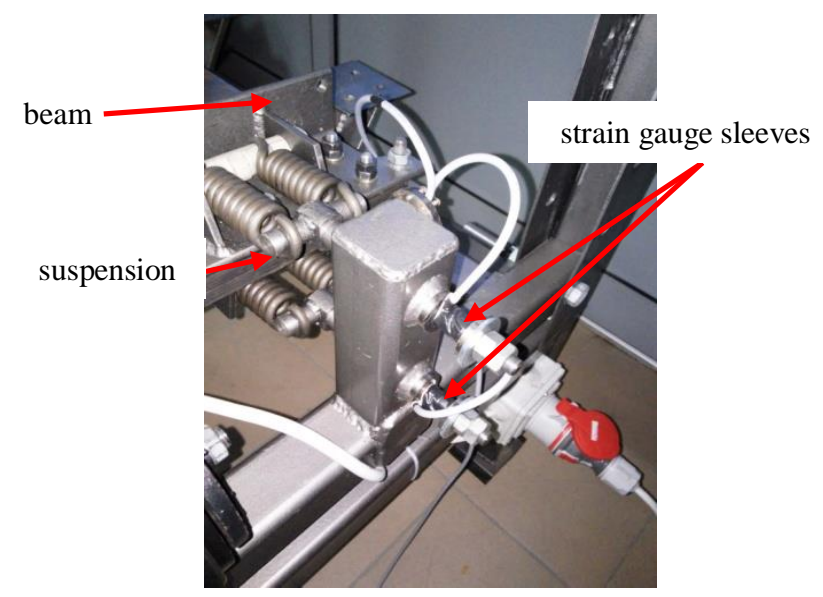

Fig. 2. The strain gauges sleeves assembly for tension force measurement

\section{EXPERIMENTAL METHODS}

As mentioned earlier, the experiment is performed for the plate without cut outs. The plate is made from $1 \mathrm{~mm}$ thickness spring steel sheet grade 1.8159 (Tab. 1).The plate is mounted between two beams of the screen with additional rubber pads for reducing plate bending stresses (Fig. 1).For estimation of tension force rough calculations were performed by using the string tension force equation:

$F_{t}=4 f^{2} l^{2} P \rho$

where: $F_{t}$ - tension force, $f$ - frequency, $l-$ string length (plate width), $P$ - string (plate) cross-section area, $\rho$ - material density.

This formula has been proposed by Slepyan and Slepyan (2014) for preliminary adjustment of screen tension to obtain the resonant frequency equal $25 \mathrm{~Hz}$. In this frequency the most efficiency screening is observed. The adjustment parameters which were applied during test are shown in Tab.2. For each adjusted tension force the resonant plate vibrations can be obtained by changing the rotational speed of electrical vibrators in the range of $2300 \div 3466 \mathrm{rpm}$. The eccentric masses are adjusted on 50\% of nominal centrifugal force equal to $2972 \mathrm{~N}$. By taking into account variable angular velocity of eccentric masses excitation force is calculated. Rotational speed data is measured by laser sensor and then converted to excitation frequency.

Tab. 1. Mechanical properties for spring steel sheet1.8159

\begin{tabular}{|l|c|}
\hline Mechanical property & Value \\
\hline Young modulus, GPa & 210 \\
\hline Ulitimate tensile stress, MPa & 590 \\
\hline Tensile Yeld stress, MPa & 395 \\
\hline Elongation $\mathrm{A}_{50}, \%$ & 24 \\
\hline Denisity, $\mathrm{g} / \mathrm{cm}^{3}$ & 7.84 \\
\hline
\end{tabular}

Tab. 2.Screen adjustment parameters

\begin{tabular}{|c|c|c|}
\hline Tension force, $\mathbf{N}$ & Excitation force, $\mathbf{N}$ & $\begin{array}{c}\text { Excitation } \\
\text { frequency, } \mathbf{H z}\end{array}$ \\
\hline 4000 & $1571 / 2151$ & 49.4 and57.8 \\
\hline 4800 & 1818 & 53.13 \\
\hline 5200 & 1913 & 54.5 \\
\hline 6000 & 1987 & 55.55 \\
\hline
\end{tabular}

Two PCB piezoelectric accelerometers with measurement range \pm 1000 gare used to determine the frequency and vibration level. Voltage signal from the sensors is converted and collected as acceleration versus time data. Further signal processing is performed in MATLAB software by using Fast Fourier Transform functions(FFT) and Chebyshev filter.

Plate vibrations are recorded also by using high speed camera with speed rate $400 \mathrm{fps}$. This rate is sufficient to determine the mode shapes of vibrating plate and to observe the system behaviour.

\section{RESULTS}

The results of the plate vibration frequency are shown in Tab. 3. The first parametric vibration is observed when the plate 
natural frequency to excitation frequency ratio is nearly equal to 0.6 in all respected cases. As the tension force increased the natural frequency for plate also increased what was expected (Fig. 3). Comparing the tension forces from rough calculations and from measurement test huge differences are observed. For this reason the equation of string tension cannot be used even for rough calculation of sieve natural vibrations. A small change of proper excitation frequency $( \pm 0.5 \mathrm{~Hz})$ caused amplitude fading. The second parametric resonance is found when the rotational speed of vibrators increases. It is obtained for plate natural frequency to excitation frequency ratio equal to 0.5 . This parametric resonance vibration was observed only for tension force equal to $4000 \mathrm{~N}$ because of the rotational speed limits of electrical vibratos. This result confirms the theory of parametric resonance that the primary parametric resonance appears when the frequency of parameter change is two times larger than the natural frequency of the system.

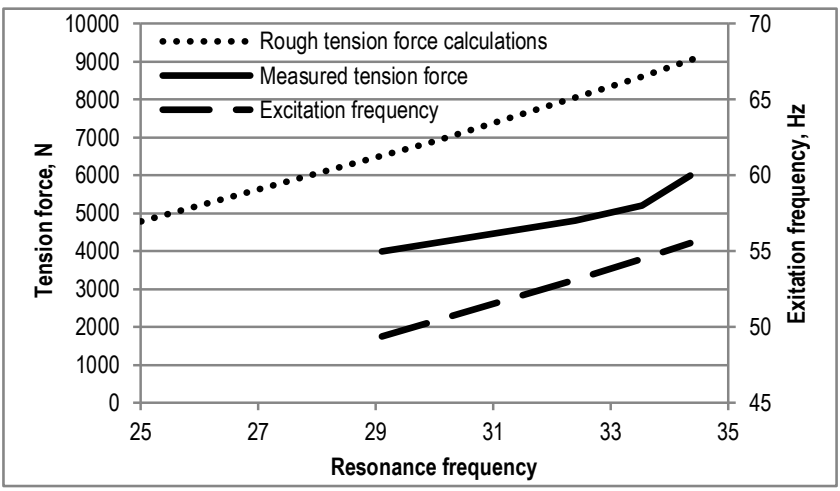

Fig. 3. Dependence among the tension force, resonance frequency and excitation frequency

Resonant frequencies of the plate are determined by analysis of the amplitude spectrum graphs (Fig.4). The highest peak in the graph demonstrates the resonant frequency of plate. In the graph for first parametric resonance there is also one high peak localized near frequency equal to $20 \mathrm{~Hz}$ (Fig.4a). This could be the effect of high amplitude screen beams vibrations which are observed during the test. Beams vibrations highly disturbed the plate motion and the screen work as well, which is confirmed by high speed camera record. For second parametric resonance there are no visible peaks close to plate resonant frequency (Fig.4b). The further peaks in high frequency level had no visible effect on plate vibrations. The screen operation is stabile and the beams vibrate with very small amplitude.

Tab. 3. Plate resonant frequencies

\begin{tabular}{|c|c|c|}
\hline $\begin{array}{c}\text { Tension force, } \\
\mathbf{N}\end{array}$ & $\begin{array}{c}\text { Plate vibration } \\
\text { frequency, } \mathrm{Hz}\end{array}$ & $\begin{array}{c}\text { Plate resonant frequency } \\
\text { lexcitation frequency ratio }\end{array}$ \\
\hline 4000 & 29.1 & 0.589 and 0.503 \\
\hline 4800 & 32.4 & 0.61 \\
\hline 5200 & 33.5 & 0.615 \\
\hline 6000 & 34.35 & 0.618 \\
\hline
\end{tabular}

Large differences between the first and the second plate parametric vibrations are clearly visible by taking into account the acceleration versus time graph (Fig. 5). The amplitude of the first resonant vibration (Fig. 5a) is less than $40 \mathrm{~g}$ and for second reso- nant vibration more than $80 \mathrm{~g}$ (Fig. 5b). Application of excitation frequency twice as large as the natural plate vibration resulted in more than twice vibration amplitude in comparison with the first parametric vibrations. This is important from the point of view of the screening efficiency. High vibration amplitude causes the vibro-impact occurrence. This phenomenon enable to screen the naturally wet fine granular materials (e.g., sand) by breaking glutted particles of material without additional water supply.

a)

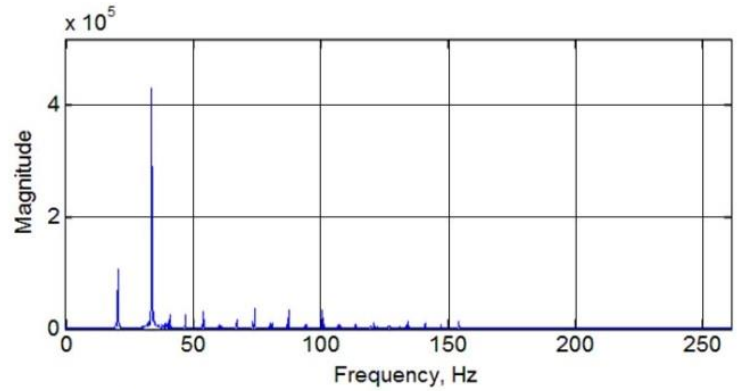

b)

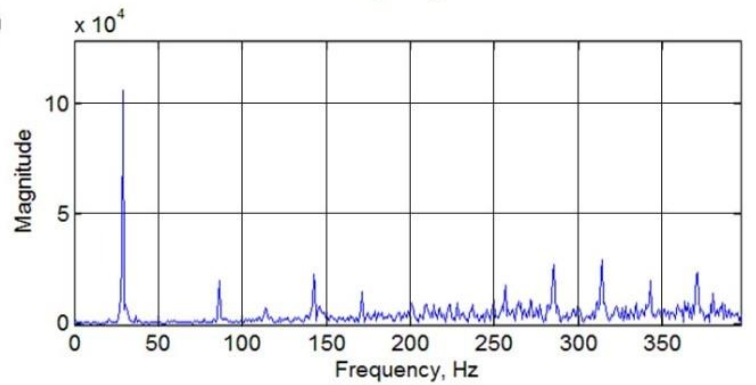

Fig. 4. Amplitude spectrum graphs: a) force tension: $5200 \mathrm{~N}$, excitation frequency: $54.5 \mathrm{~Hz}$; b) force tension: $4000 \mathrm{~N}$, excitation frequency: $57.8 \mathrm{~Hz}$

a)

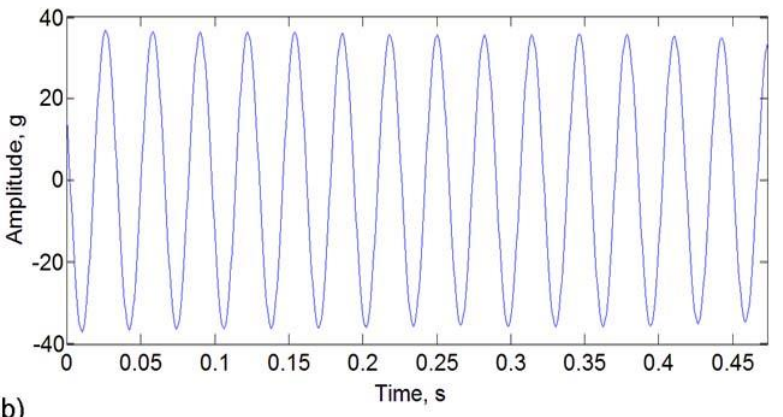

b)

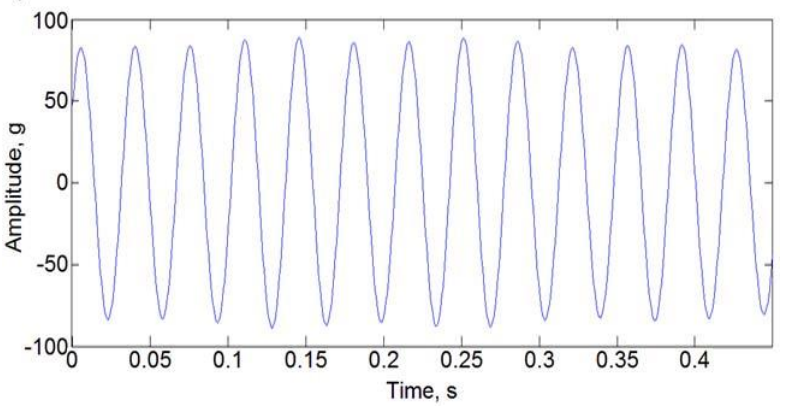

Fig. 5. Filtered acceleration versus time data for force tension $4000 \mathrm{~N}$, a) excitation frequency: $49.4 \mathrm{~Hz}$; b) excitation frequency: $57.8 \mathrm{~Hz}$

Beside the vibrations amplitude level the mode shape of vibration is also very important. The first mode shape of plate vibration is observed in both excitation frequencies $49.4 \mathrm{~Hz}$ and $57.8 \mathrm{~Hz}$ 
(Fig. 6). This confirms that the first natural frequency of a plate with tension equal to $4000 \mathrm{~N}$ is $29.1 \mathrm{~Hz}$. For second parametric vibration large plate deformation is visible caused by large vibration amplitude (Fig. 6b).
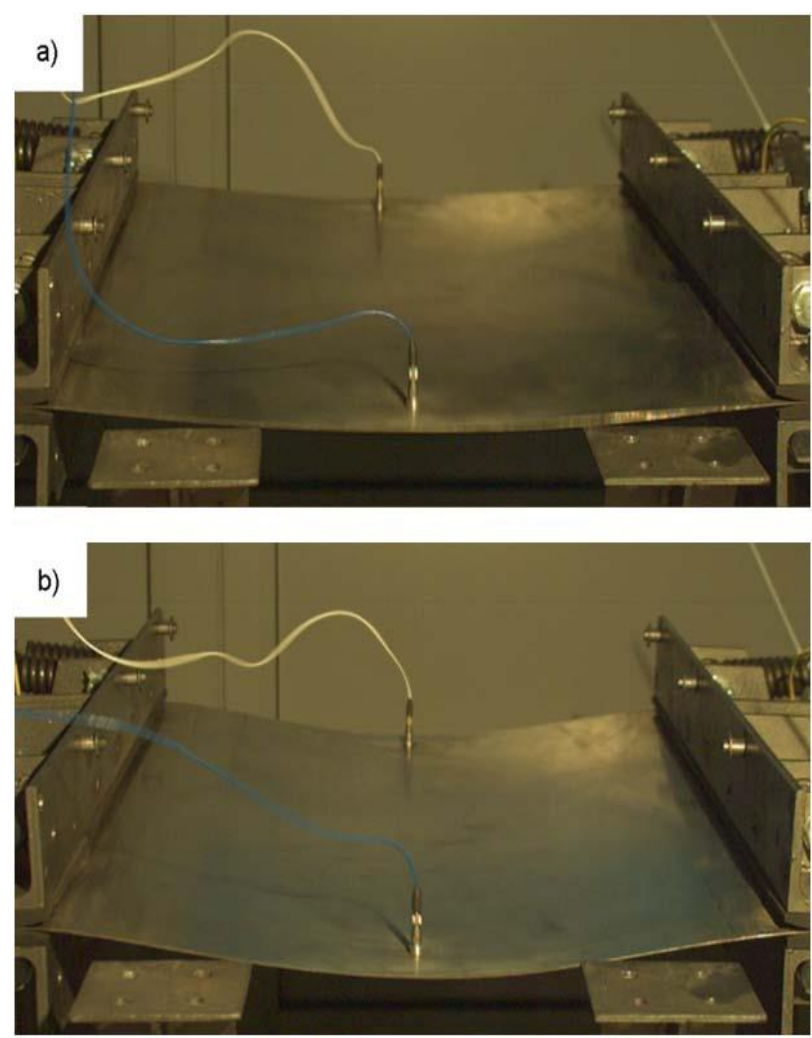

Fig. 6. Plate vibrations shapes (maximum deformation) for tension force $4000 \mathrm{~N}$ caught by high speed camera: excitation frequency: $49.4 \mathrm{~Hz}$; b) excitation frequency: $57.8 \mathrm{~Hz}$

\section{CONCLUSIONS}

This work deals with the experimental investigations of the laboratory screen operating in the parametric resonance condition.

In the experimental investigation, natural frequency of the sieve plate and parameter modulation frequency are determined for four cases of the tension force value. As it was expected the value of the sieve plate natural frequency and parameter modulation frequency grow parallel to the growing tension force.

The plate vibrations in parametric resonance conditions occurred for two resonant frequency to excitation frequency ratios 0.6 and 0.5.Plate parametric vibrations for frequency ratio 0.6 characterise relatively low amplitude. High amplitude beams vibrations with frequency of $20 \mathrm{~Hz}$ were observed which disturbed the plate vibrations. The highest sieve plate amplitude is obtained when the parameter modulation frequency is two times larger than the natural frequency of the sieve plate.

The experimental approach demonstrated in this paper can be used for the case with the real sieve and for further investigation related to the screening process.

\section{REFERENCES}

1. Başar Y., Eller C., Krätzig W.B. (1987), Finite element procedures for parametric resonance phenomena of arbitrary elastic shell structures, Computational Mechanics, 2, 89-98.

2. Bąk Ł., Noga S., Skrzat A., Stachowicz F. (2013), Dynamic analysis of vibrating screener system, Journal of Physics: Conference Series, 451, 012028.

3. de Silva C. (2005), Vibration and shock handbook, Taylor \& Francis, Boca Raton.

4. Hagedorn P., Koval L.R. (1971), On the parametric stability of a Timoshenko beam subjected to a periodic axial load, Ingenieur Archiv, 40, 211-220.

5. He X.-M., Liu Ch.-S. (2009), Dynamics and screening characteristics of vibrating screen with variable eliptical trace, Mining Science and Technology, 19, 0508-0513.

6. Kaliski S. (1996), Vibrations and waves in solids, IPPT PAN, Warsaw (in Polish).

7. Li Z. (1995), Chaotic vibration sieve, Mechanism and Maschine Theory, 30, 613-618.

8. Michalczyk J. (1995), Vibrating machinery, dynamic calculatons, vibration and noise, WNT, Warsaw (in Polish).

9. Nguyen H. (2013), Simultaneous resonances involving two mode shapes of parametrically-excited rectangular plates, Journal of Sound and Vibration, 332, 5103-5114.

10. Osiński J. (1985), The analysis of parametric vibrations of continuous systems with constant transversal loading by asymptotic method and finite elements, Journal of Theoretical and Applied Mechanics, Vol. 2, No. 23, 241-254 (in Polish).

11. Parszewski Z. (1982), Vibration and dynamics of machines, WNT, Warsaw (in Polish).

12. Slepyan L.I., Slepyan V.I. (2013), Modeling of parametrically excited vibrating, Journal of Physics: Conference Series, 451, 012026.

13. Slepyan L.I., Slepyan V.I. (2014), Coupled mode parametric resonance in a vibrating screen model, Mechanical Systems and Signal Processing, 43, 295-304.

14. Song Y., Jiang X.-H., Song J., Zhang J.-X. (2009), Dynamic analysis of a chaotic vibrating screen, Procedia Earth and Planetary Science, 1, 1525-1531.

15. Virgin L.N. (2007), Vibration of axially loaded structures, Cambridge University Press, Cambridge.

16. Yang X.-D., Chen L.-Q. (2006), Stability in parametric resonance of axially accelerating beams constituted by Boltzmann's superposition principle, Journal of Sound and Vibration, 289, 54-65.

17. Zhao Y.-M., Liu Ch.-S., He X.-M., Zhang Ch.-Y., Wang Y.-B., Ren Z.-T. (2009), Dynamic design theory and application of large vibrating screen, Procedia Earth and Planetary Science, 1, 776-784.

Acknowledgments: This work was supported by the European Research Agency - 7th FP PEOPLE PROGRAMME Marie Curie Industry-Academia Partnerships and Pathways, grant agreement No. 284544 